\title{
http://revistainvestigacionacademicasinfrontera.com
}

\section{Análisis comparativos del ranking de pobreza en los municipios del Estado de Sonora, medidos con los modelos de CONEVAL y CONAPO, 2010.}

Dr. Juan José García Ochoa, Dra. Leticia González Velásquez Dr. Iván Ochoa Vázquez’ Dra. Adriana Leticia Navarro Verdugo ${ }^{1}$, Dr. Modesto Barrón Wilson ${ }^{1} \&$ Dr. Ignacio Yocupicio Villegas.

Universidad de Sonora

\section{Resumen}

Se analizan los niveles de pobreza para 74 entidades conformadas por los 72 municipios, el Estado de Sonora en su conjunto y el país México, utilizando para ello los modelos de medición de la pobreza desarrollados tanto por el CONEVAL como por CONAPO. Los datos del primer modelo son analizados mediante estadística univariada, con la que se identifican los rankings y porcentaje de pobreza, los porcentajes de la población con ingresos en línea de bienestar mínima, línea de bienestar económica y el porcentaje de la población con al menos una de seis carencias sociales. Los resultados de ambos modelos coinciden en identificar a 6 de los 10 municipios más pobres de Sonora en lo general estos son: Yécora, Álamos, Rosario, San Miguel de Horcasitas, San Ignacio Río Muerto y Etchojoa.

Palabras claves: indicadores de pobreza, pobreza extrema, pobreza moderada

Comparative analysis of the poverty ranking in the municipalities of the State of Sonora, measured with the CONEVAL and CONAPO models, 2010. 
Año 10.

Revista de Investigación

Núm. 26

Académica sin Frontera

8870

ISSN: 2007 .

\section{http://revistainvestigacionacademicasinfrontera.com}

\section{Summary}

The poverty levels are analyzed for 74 entities formed by the 72 municipalities, the State of Sonora as a whole and Mexico, using the poverty measurement models developed by CONEVAL and CONAPO. The data of the first model are analyzed using univariate statistics, which identify the rankings and percentage of poverty, the percentages of the population with minimum well-being online income, economic welfare line and the percentage of the population with at least one Of six social deficiencies. The results of both models coincide in identifying 6 of the 10 poorest municipalities of Sonora in general these are: Yécora, Álamos, Rosario, San Miguel de Horcasitas, San Ignacio Rio Muerto and Etchojoa.

Keywords: indicators of poverty, extreme poverty, moderate poverty

\section{Introducción}

En el sentido de Sen (2000), (citado por Robles et al. 2011), plantea que se deben reconocer las libertades fundamentales del hombre dentro del desarrollo social. Para ello las define como: la capacidad de satisfacer las necesidades básicas, el desarrollo integral del individuo en su desarrollo físico e intelectual y el derecho de cada persona de vivir de forma sana tanto física como emocional, en una sociedad donde pueda expresarse y participar abierta y activamente. Se incluye además al Estado, la obligación de satisfacer estas necesidades dentro de su plan de desarrollo y en el presupuesto nacional (Robles I., Borbón S., Medellín P., \& Calles M., 2011).

Con las aportaciones de Sen (2000), se ha desarrollado entre otros modelos, el del Programa de las Naciones Unidas para el Desarrollo (PNUD), concebido como un modelo para buscar acciones de colaboración para que el Estado implemente una estrategia de desarrollo mediante la creación de políticas públicas que contribuyan a la solución priorizada de quienes experimenten la mayor exclusión y desigualdad de oportunidades y con ello erradicar la pobreza extrema, contener y hacer retroceder la desigualdad, y lograr el acceso universal a servicios básicos, de tal modo que todas las personas tengan un nivel mínimo de bienestar. Mediante la investigación en un índice de desarrollo 


\section{http://revistainvestigacionacademicasinfrontera.com}

humano (IDH), conformado por dos pilares: el primero que es el social, el cual a su vez está formado por cuatro variables 1) esperanza de vida al nacer infantil, 2) años promedio de escolaridad, 3) años esperados de escolaridad y 4) índice combinado de educación; el segundo pilar es el económico que está formado por una sola variable que es el PIB per cápita medido en dólares estadounidenses (PNUD, 2014).

Por su parte, el Consejo Nacional de Población (CONAPO), a inicios de la década de los noventas, emprendió el proyecto "Desigualdad regional y marginación municipal en México", cuyos resultados del primer informe, se publicaron en el libro Indicadores socioeconómicos e índice de marginación, 1990. A lo largo de este cuarto de siglo se ha madurado una nueva visión que ubica al desarrollo económico en forma sistémica, ya no sólo es la idea de promover el crecimiento económico, sino de considerar la calidad y las condiciones de vida de las personas y comunidades. El índice de marginación está concebido con el interés particular de ser una medida que dé cuenta de las carencias que padece la población. La marginación expresa la falta de oportunidades sociales para los ciudadanos, sus familias y comunidades exponiéndolas a privaciones, riesgos y vulnerabilidades sociales, cuya reversión requiere de la intervención de los agentes públicos, privados, sociales e institucionales. La estimación del índice de marginación se logra gracias al modelo conformado por tres dimensiones, las cuales miden los aspectos de carencia social relacionada con la educación, calidad de vivienda y la de ingresos económicos; y una cuarta dimensión que mide la distribución de la población. Para ello estas dimensiones son aproximadas mediante 9 variables operativas, las cuales son recabadas del Censo de Población y Vivienda, el muestreo censal y la Encueta Nacional de Ocupación y Empleo (ENOE) (CONAPO, 2010).

La relación entre pobreza y evaluación de programas sociales hacen necesario recurrir al trabajo de Sen (2000), quien señala que se deben satisfacer los derechos sociales básicos y de ingreso del individuo que le permita una vida digna. En este contexto, la medición de la pobreza puede ser concebida como una evaluación global de las políticas de desarrollo social, la cual permite evaluar, en el mediano y largo plazo, el desempeño de 


\section{http://revistainvestigacionacademicasinfrontera.com}

las políticas de superación de la pobreza. Para ello la Ley General de Desarrollo Social (LGDS), fue aprobada en las cámaras de Diputados y de Senadores, y promulgada el 20 de enero de 2004, tiene como objetivo central “... garantizar el pleno ejercicio de los derechos sociales consagrados en la Constitución Política de los Estados Unidos Mexicanos, asegurando el acceso de toda la población al desarrollo social”. Es decir, que por ley se promueve las condiciones que aseguren el disfrute de los derechos sociales y económicos con sentido social que eleve el ingreso de la población y contribuya a reducir la desigualdad (CONEVAL, 2014).

Además, la LGDS establece que el Consejo Nacional de Evaluación de la Política de Desarrollo Social (CONEVAL), sea la institución encargada de evaluar la política y programas de desarrollo social y también sea la que emita los lineamientos y criterios para la definición, identificación y medición de la pobreza. En este sentido, para medir la pobreza en México el CONEVAL ha definido la pobreza multidimensional como:

"Una persona se encuentra en situación de pobreza multidimensional cuando no tiene garantizado el ejercicio de al menos uno de sus derechos para el desarrollo social, y si sus ingresos son insuficientes para adquirir los bienes y servicios que requiere para satisfacer sus necesidades."

La definición anterior contiene dos pilares: el primero relacionada con el social que identifica a la población con al menos una carencia social, de entre las seis variables operativas identificadas y, la segunda relacionada con el económico y sus dos variables operativos del ingreso para satisfacer sus necesidades (CONEVAL, 2014).

\section{Material y método}

Diseño de la investigación. El tipo de estudio fue de diseño no experimental de clasificación transeccional, y dado que por su naturaleza de describir situaciones específicas de los municipios, el nivel de relación entre las variables interdependientes con la variable de respuesta obtenida, se consideró a su vez como un diseño de investigación causal (Hernández Sampieri, Fernández Collado, \& Baptista Lucio, 2010). 


\section{http://revistainvestigacionacademicasinfrontera.com}

Objeto de estudio y selección de la muestra. Los resultados de la investigación corresponden a los 72 municipios, los cuales forman parte del estado de Sonora con quien se contrastan y éstos a su vez son contrastados a nivel del país.

Fuentes de información secundaria. Las fuentes de información que nutrieron este trabajo, son los bancos de datos del CONEVAL y de CONAPO.

Elaboración y aplicación del instrumento. Se utilizó el modelo teórico de medición multidimensional de la pobreza en México de CONEVAL, el cual se presenta en el cuadro 1; y de CONAPO, mostrado en el cuadro 2. Los datos recopilados fueron cargados a una hoja de datos de Microsoft Office Excel 2007, previamente adecuada en base a los requerimientos del modelo teórico y posteriormente fueron procesados en los paquetes estadísticos SPSS V.21 y Minitab V.17.

Cuadro 1. Dimensiones e indicadores de pobreza, en México.

\begin{tabular}{|l|l|}
\hline $\begin{array}{l}\text { Variable global } \\
\text { dependiente }\end{array}$ & \multicolumn{1}{c|}{ Componentes de la variable dependiente } \\
\hline Pobreza & $\begin{array}{l}\text { Población no pobre por ingresos y no vulnerable por carencias } \\
\text { sociales (cuadrante I) }\end{array}$ \\
\hline
\end{tabular}




\section{http://revistainvestigacionacademicasinfrontera.com}

\begin{tabular}{|l|l|}
\hline & $\begin{array}{l}\text { Población vulnerable solo por carencias sociales (cuadrante II) } \\
\text { Población en situación de pobreza (cuadrante III) } \\
\quad \quad \text { Población en situación de pobreza moderada } \\
\text { Población en situación de pobreza extrema }\end{array}$ \\
\hline $\begin{array}{l}\text { Variable } \\
\text { independiente }\end{array}$ & \multicolumn{1}{|c|}{ Componentes de la variable independiente } \\
\hline Carencias Sociales & $\begin{array}{l}\text { Rezago educativo } \\
\text { Acceso a los servicios de salud } \\
\text { Acceso a la seguridad social } \\
\text { Calidad y espacios de la vivienda } \\
\text { Accesos a los servicios básicos en la vivienda } \\
\text { Acceso a la alimentación }\end{array}$ \\
\hline Ingresos & $\begin{array}{l}\text { Población con un ingreso inferior a la línea de bienestar mínimo } \\
\text { (LBM). } \\
\text { Población con un ingreso inferior a la línea de bienestar } \\
\text { económico (LBE). }\end{array}$ \\
\hline
\end{tabular}

Fuente: elaboración propia con información de CONEVAL, 2010.

Cuadro 2. Dimensiones e indicadores para medir el índice de marginación, en México.

\begin{tabular}{|l|l|}
\hline $\begin{array}{l}\text { Dimensión } \\
\text { socioeconómicas }\end{array}$ & \multicolumn{1}{|c|}{ Indicadores para medir la intensidad de la exclusión } \\
\hline Educación & $\begin{array}{l}\text { 1.- Porcentaje de la población de 15 años o más analfabeta } \\
\text { 2.- Porcentaje de la población de 15 años o más sin primaria } \\
\text { completa }\end{array}$ \\
\hline Vivienda & $\begin{array}{l}\text { 1.- Porcentaje de ocupantes en viviendas sin drenaje ni excusado } \\
\text { 2.- Porcentaje de ocupantes en viviendas sin energía eléctrica } \\
\text { 3.- Porcentaje de ocupantes en viviendas sin agua entubada } \\
\text { 4.- Porcentaje de viviendas con algún nivel de hacinamiento } \\
\text { 5.- Porcentaje de ocupantes en viviendas con piso de tierra }\end{array}$ \\
\hline Distribución de la & $\begin{array}{l}\text { 1.- Porcentaje de la población en localidades con menos de 5000 } \\
\text { (cinco mil) habitantes. }\end{array}$ \\
\hline población & $\begin{array}{l}\text { 1.- Porcentaje de la población ocupada con ingresos de hasta } 2 \\
\text { (dos), salarios mínimos. }\end{array}$ \\
\hline monetarios & \\
\hline
\end{tabular}

Fuente: elaboración propia con información de CONAPO, 2010. 


\section{http://revistainvestigacionacademicasinfrontera.com}

\section{Resultados y Discusión}

Desde la definición de pobreza que da el CONEVAL, se puede ver que esta depende de dos variables: la que es debida a los ingresos y la que es debida a las carencias sociales. A su vez la variable ingresos, se subdivide en los ingresos en la LBM y los que están en la LBE. Por su parte, los del tipo LBM se refieren al porcentaje de la población que solo cuenta con los ingresos para abastecerse de una canasta alimentaria por $\$ 613.8$ pesos semanales si son del área rural y por \$ 874.63 pesos si son del área urbana; mientras que los pobres que están en la LBE cuentan además con un ingreso por \$589 pesos para gastos de una canasta no alimentaria si son del área rural y por \$1,047.11 pesos si son del área urbana, es decir, la población que está en la LBE cuenta con una canasta total por $\$ 1,202.80$ pesos semanales si son de área rural y una por $\$ 1,921.74$ si son del área urbana. Continuando con la definición, la pobreza además se mide considerando a toda aquella población que carece de al menos uno de las seis carencias sociales identificadas en el modelo, por lo tanto, la pobreza total debe ser aquella que integre tanto al porcentaje de población con carencias económicas como sociales, sin embargo, el cálculo de la pobreza que estima el CONEVAL, solo está referida a la pobreza del cuadrante III, es decir, como la suma de pobreza moderada y la de pobreza extrema que se relacionan con los ingresos y las de carencia social, mientras que los cuadrantes II y IV son mostradas como indicadores independientes que también estiman a la pobreza pero de manera separada. Por lo anterior, y para ser congruente con el análisis del CONEVAL el modelo conceptual con las ocho variables identificadas fue analizado mediante estadística univariada, para conocer los niveles de pobreza como variable de respuesta, los resultados indican que los diez municipios más pobres de Sonora en orden decreciente son: Yécora, San Miguel de Horcasitas, Álamos, Tubutama, Nácori Chico, Rosario, Benito Juárez, San Ignacio Río Muerto, Etchojoa y Tepache. Es de notarse que Yécora tiene el 74\% de pobreza, el $46.6 \%$ de su población están con ingresos en LBM, el 80.5\% de la población están con ingresos en LBE y el $96.1 \%$ de su población tienen al menos una de las seis carencias sociales que 
Año 10.

Revista de Investigación

Núm. 26

Académica sin Frontera

8870

ISSN: 2007 -

\section{http://revistainvestigacionacademicasinfrontera.com}

considera el modelo CONEVAL. Los detalles del análisis para el resto de las entidades y sus variables críticas, se presentan en el cuadro 3 del anexo.

Por otro lado, de acuerdo con el modelo y los datos reportados de CONAPO, estos al ser analizadas con la metodología de estadística multivariada por componentes principales, (y no por estadística univariada como lo hace CONEVAL), los resultados indican que los diez municipios más pobres de Sonora en una escala de cero a cien, ordenados de manera decreciente son: Quiriego, Álamos, Rosario, Yécora, San Miguel de Horcasitas, Etchojoa, Cucurpe, San Ignacio Río Muerto, La Colorada y Bacanora. Por el contrario, los diez municipios menos pobres son: Cananea, Nacozari de García, Naco, Hermosillo, Magdalena, Moctezuma, Santa Ana, Cajeme, Fronteras y Agua Prieta. La información completa, se encuentra en el cuadro 4.

El lector alerta habrá notado que las diferencias de los resultados se deben a que la información analizada se hacen con diferentes métodos estadísticos (univariado y multivariados) y que demás tanto los modelos como sus variables son también diferentes. Más aun, usted puede llegar a resultados en términos generales similares, si se enfoca en solo una, dos o más de las variables reportadas en el cuadro 5.

\section{Conclusiones}

El modelo de medición multidimensional de la pobreza que ha desarrollado el CONEVAL, no explica en su metodología como calcula la pobreza, la pobreza extrema y la pobreza moderada, sin embargo, desde su definición de pobreza se puede deducir que esta se calcula en función de una variable acumulativa que considere al menos una de las carencias sociales y otra que considere la población con ingresos insuficientes para adquirir los bienes y servicios básicos, es decir, se estaría considerando a toda la población que se encuentra en los cuadrantes II, III y IV del modelo.

Por otra parte, el CONEVAL menciona que, la pobreza del cuadrante III se desglosa en pobreza extrema y pobreza moderada, la primera (la extrema) se refiere al porcentaje de 


\section{http://revistainvestigacionacademicasinfrontera.com}

la población urbana o rural que solo cuenta con los ingresos para abastecerse de una canasta básica alimentaria y que esta población se encuentra en la LBM; mientras que los pobres moderados cuentan además con un ingreso adicional para sufragar los gastos de servicios no alimentarios que sumados a los ingresos de la canasta básica alimentaria, entonces estaríamos hablando de la población que está en la LBE, que cuenta con una canasta total por $\$ 1,202.80$ pesos semanales si son de área rural $\mathrm{y}$, una por $\$ 1,921.74$ si son del área urbana. Sin embargo, para estar de acuerdo con la definición de pobreza, se debe incluir a los pobres que estén además en el cuadrante II y en el cuadrante III en un mismo análisis de pobreza global. Lo que significaría que municipios como Yécora, S.M. de Horcasitas y Tubutama tendrían al menos un $96.1 \%$, 94.8\% y $95.3 \%$ de su población en condiciones de pobreza. Es decir, solo se está estimando como pobreza a la población que se encuentra en el cuadrante III, y el resto de los indicadores solo identifican por separado su contribución de la pobreza en los cuadrantes II y IV. Aun así, todas las entidades mostraron altos porcentajes de pobreza en su población, sin embargo, para los municipios de Hermosillo, Nacozari de García, Cajeme, Huépac y Guaymas, mostraron niveles por debajo del 30\%, colocándose éstas en los "mejores" cinco lugares, mientras que el Estado de Sonora tiene un 33.8\% (ranking 9) y el país un 46.3\% (ranking 39).

Ambos modelos CONEVAL y CONAPO, coinciden en la identificación en 6 de los 10 diez municipios más pobres, estos son en lo general a: Yécora, Álamos, Rosario, San Miguel de Horcasitas, San Ignacio Río Muerto y Etchojoa. Por el contrario, los modelos solo coinciden en identificar a 3 de los 10 municipios menos pobres, dichos municipios son: Hermosillo, Nacozari de García y Cajeme.

\section{Referencias}

CONAPO. (2010). Indice de marginación por entidad federativa y municipio. México, D.F.: Consejo Nacional de Población.

CONEVAL. (2012). Informe de Pobreza en México 2010: el país, los estados y sus municipios. México, D.F.: Impresora y Encuadernadora Progreso S.A. de C. V. (IEPSA). 


\section{http://revistainvestigacionacademicasinfrontera.com}

CONEVAL. (2014). Metodología para la medición multidimensional de la pobreza en México (segunda edición). México: talleres Gráficos de México, Canal del Norte No. 80.

Garcia Ochoa, J. J., Ochoa Vázquez, I., González Velásquez, L. M., \& Mendoza León, J. G. (26 de Julio-Diciembre de 2015). Ranking de pobreza en los municipios del Estado de Sonora, medidos con el modelo de CONEVAL, 2010. Revista de Investigación Académica sin Frontera(21), 1-10. Obtenido de http://revistainvestigacionacademicasinfrontera.com

Garza Cantú, V., Polendo Garza, J., \& García Hernández, F. (2005). Los estados mexicanos. Sus activos y su dinamismo económico y social. México: Miguel Angel Porrúa.

Hernández Sampieri, R., Fernández Collado, C., \& Baptista Lucio, P. (2010). Metodología de la investigación (5a ed.). Perú: McGraw-Hill.

IMCO. (2007). Competitividad Urbana 2007. México: Instituto Mexicano para la Competitividad A.C.

PNUD. (2014). Indice de Desarrollo Humano Municipal en México: nueva metodología. México, DF: Danda Diseño e Impresión.

Robles I., J. C., Borbón S., R. R., Medellín P., A., \& Calles M., F. (2011). Desarrollo, innovación, organización y gestión socioeconómica del Noroeste de México. México: PEARSON. 


\section{http://revistainvestigacionacademicasinfrontera.com}

\section{Anexo}

Cuadro 3 Ranking ordenado de mayor a menor pobreza global, 2010

\begin{tabular}{|c|c|c|c|c|c|}
\hline \multirow{3}{*}{$\begin{array}{l}\text { Economía de la } \\
\text { entidad. }\end{array}$} & \multicolumn{5}{|c|}{ Grado de pobreza para 74 entidades } \\
\hline & \multicolumn{2}{|c|}{ Pobreza } & \multirow{2}{*}{$\begin{array}{l}\text { Porcentaje de } \\
\text { población con } \\
\text { Ingresos en } \\
\text { LBM }\end{array}$} & \multirow{2}{*}{$\begin{array}{c}\text { Porcentaje de } \\
\text { población con } \\
\text { Ingresos en LBE }\end{array}$} & \multirow{2}{*}{$\begin{array}{c}\text { \% de población con } \\
\text { al menos } 1 \text { de } 6 \\
\text { carencias sociales }\end{array}$} \\
\hline & Ranking & $\%$ & & & \\
\hline Yécora & 74 & 78,7 & 46,6 & 80,5 & 96,1 \\
\hline S. M. de Horc. & 73 & 71,2 & 48,0 & 73,7 & 94,8 \\
\hline Álamos & 72 & 65,0 & 33,3 & 66,4 & 92,0 \\
\hline Tubutama & 71 & 63,2 & 31,9 & 64,4 & 95,3 \\
\hline Nácori Chico & 70 & 61,4 & 27,1 & 62,8 & 93,1 \\
\hline Rosario & 69 & 61,3 & 26,7 & 66,5 & 88,3 \\
\hline Benito Juárez & 68 & 59,6 & 27,3 & 63,5 & 86,7 \\
\hline San I. Río M. & 67 & 59,4 & 24,9 & 63,4 & 88,9 \\
\hline Etchojoa & 66 & 57,6 & 25,3 & 61,1 & 87,0 \\
\hline Tepache & 65 & 56,4 & 25,2 & 58,8 & 88,4 \\
\hline Bavispe & 64 & 56,4 & 22,4 & 57,5 & 94,7 \\
\hline Onavas & 63 & 56,1 & 22,2 & 59,8 & 90,0 \\
\hline Trincheras & 62 & 55,6 & 24,8 & 58,7 & 91,3 \\
\hline Bacadéhuachi & 61 & 54,9 & 23,6 & 56,9 & 90,2 \\
\hline Sahuaripa & 60 & 54,7 & 22,2 & 59,5 & 84,8 \\
\hline Sáric & 59 & 53,7 & 22,4 & 54,2 & 96,5 \\
\hline Bacanora & 58 & 53,6 & 22,4 & 55,8 & 89,6 \\
\hline Gral. P.E. C. & 57 & 53,3 & 27,7 & 56,8 & 89,5 \\
\hline Bacerac & 56 & 53,3 & 18,4 & 54,8 & 89,6 \\
\hline Carbó & 55 & 53,0 & 30,4 & 57,5 & 88,1 \\
\hline Huatabampo & 54 & 52,7 & 20,8 & 56,7 & 84,9 \\
\hline Santa Cruz & 53 & 52,7 & 20,0 & 54,1 & 93,4 \\
\hline Rayón & 52 & 52,1 & 23,4 & 54,3 & 89,2 \\
\hline Opodepe & 51 & 52,0 & 23,1 & 55,1 & 89,0 \\
\hline Huachinera & 50 & 51,8 & 20,8 & 52,8 & 93,5 \\
\hline Suaqui Grande & 49 & 51,6 & 19,2 & 54,5 & 91,1 \\
\hline Naco & 48 & 50,8 & 17,7 & 55,0 & 86,7 \\
\hline Cucurpe & 47 & 50,6 & 18,6 & 52,8 & 91,6 \\
\hline La Colorada & 46 & 50,2 & 21,7 & 53,2 & 91,4 \\
\hline Arizpe & 45 & 49,8 & 20,4 & 52,6 & 88,1 \\
\hline Soyopa & 44 & 49,4 & 18,3 & 53,0 & 84,6 \\
\hline Arivechi & 43 & 49,2 & 19,6 & 52,4 & 85,7 \\
\hline Atil & 42 & 47,8 & 22,7 & 52,1 & 82,8 \\
\hline Altar & 41 & 47,7 & 23,8 & 51,8 & 87,3 \\
\hline Villa Pesqueira & 40 & 47,3 & 15,7 & 49,9 & 89,3 \\
\hline E.U. Mex. & 39 & 46,3 & 19,4 & 52,0 & 75,0 \\
\hline
\end{tabular}




\section{http://revistainvestigacionacademicasinfrontera.com}

Continuación del cuadro 3. Ranking ordenado de mayor a menor pobreza global

\begin{tabular}{|c|c|c|c|c|c|}
\hline \multirow{3}{*}{$\begin{array}{c}\text { Economía de } \\
\text { la entidad }\end{array}$} & \multicolumn{5}{|c|}{ Grado de pobreza para 74 entidades } \\
\hline & \multicolumn{2}{|c|}{ Pobreza } & \multirow{2}{*}{$\begin{array}{c}\text { Porcentaje de } \\
\text { población con } \\
\text { Ingresos en LBM }\end{array}$} & \multirow{2}{*}{$\begin{array}{c}\text { Porcentaje de } \\
\text { población con } \\
\text { Ingresos en LBE }\end{array}$} & \multirow{2}{*}{$\begin{array}{c}\text { \% de población } \\
\text { con al menos } 1 \\
\text { de } 6 \text { carencias } \\
\text { sociales }\end{array}$} \\
\hline & Ranking & $\%$ & & & \\
\hline Agua Prieta & 38 & 46,2 & 17,7 & 53,8 & 74,6 \\
\hline $\begin{array}{l}\text { San P. de la } \\
\text { Cueva }\end{array}$ & 37 & 45,3 & 15.1 & 47.4 & 89.3 \\
\hline Bácum & 36 & 44,5 & 14,4 & 51,1 & 80,8 \\
\hline Imuris & 35 & 44,0 & 14,0 & 47,9 & 84,5 \\
\hline Puerto Peñasco & 34 & 43,7 & 16,1 & 50,2 & 76,2 \\
\hline Divisaderos & 33 & 42,3 & 13,0 & 46,3 & 83,0 \\
\hline Quiriego & 32 & 41,7 & 17,8 & 42,7 & 94,8 \\
\hline Ures & 31 & 41,5 & 13,5 & 46,7 & 79,5 \\
\hline Aconchi & 30 & 41,4 & 14,2 & 44,9 & 83,0 \\
\hline Banámichi & 29 & 41,3 & 15,0 & 44,9 & 82,2 \\
\hline Fronteras & 28 & 40,9 & 18,2 & 56,3 & 64,2 \\
\hline Magdalena & 27 & 40,8 & 13,4 & 48,7 & 71,8 \\
\hline Bacoachi & 26 & 40,7 & 12,5 & 43,1 & 88,4 \\
\hline Baviácora & 25 & 40,1 & 13,4 & 44,6 & 82,2 \\
\hline Benjamín H. & 24 & 39,9 & 13,9 & 50,0 & 68,9 \\
\hline Cananea & 23 & 39,6 & 13,1 & 46,7 & 75,2 \\
\hline San Luis R.C & 22 & 38,8 & 10,9 & 43,3 & 77,6 \\
\hline Caborca & 21 & 38,7 & 12,1 & 45,5 & 72,6 \\
\hline Mazatán & 20 & 38,1 & 11,3 & 41,0 & 81,9 \\
\hline Oquitoa & 19 & 37,0 & 15,0 & 41,8 & 81,4 \\
\hline Huásabas & 18 & 37,0 & 11,5 & 41,3 & 79,1 \\
\hline Pitiquito & 17 & 36,2 & 10,7 & 42,6 & 69,3 \\
\hline Santa Ana & 16 & 36,2 & 10,5 & 42,5 & 72,3 \\
\hline Granados & 15 & 36,0 & 10,9 & 39,4 & 80,3 \\
\hline San F. de J. & 14 & 35,6 & 11,6 & 40,1 & 75,6 \\
\hline Navojoa & 13 & 34,8 & 10,5 & 40,2 & 71,0 \\
\hline Cumpas & 12 & 34,5 & 10,2 & 41,7 & 69,7 \\
\hline Moctezuma & 11 & 34,4 & 9,9 & 41,5 & 67,7 \\
\hline Nogales & 10 & 34,1 & 10,2 & 41,1 & 66,7 \\
\hline Sonora & 9 & 33,8 & 11,1 & 40,2 & 82,1 \\
\hline Empalme & 8 & 33,4 & 11,2 & 40,8 & 67,0 \\
\hline Villa Hidalgo & 7 & 33,1 & 9,4 & 39,3 & 72,1 \\
\hline San Javier & 6 & 30,3 & 8,6 & 32,0 & 93,5 \\
\hline Guaymas & 5 & 28,9 & 9,2 & 36,3 & 59,1 \\
\hline Huépac & 4 & 26,7 & 7,5 & 32,2 & 69,4 \\
\hline Cajeme & 3 & 26,6 & 7,4 & 34,0 & 58,1 \\
\hline $\begin{array}{l}\text { Nacozari de } \\
\text { García }\end{array}$ & 2 & 26,5 & 10,0 & 39,7 & 49,8 \\
\hline Hermosillo & 1 & 25,4 & 7,2 & 32,2 & 57,5 \\
\hline
\end{tabular}

Fuente: elaboración propia con datos del CONEVAL

Cuadro 4 Ranking ordenado de mayor a menor pobreza global, 2010. 
http://revistainvestigacionacademicasinfrontera.com

\begin{tabular}{|c|c|c|c|c|c|c|c|c|c|c|c|c|}
\hline Entidad de analisis & $\begin{array}{c}\text { Población } \\
\text { Total } \\
\end{array}$ & $\begin{array}{c}\text { \% Pob. } \\
\text { Analfabeta } \\
\text { de } 15 \text { años o } \\
\text { más }\end{array}$ & $\begin{array}{c}\% \text { Pob. Sin } \\
\text { primaria } \\
\text { completa de } \\
15 \text { años o } \\
\text { más }\end{array}$ & $\begin{array}{l}\text { \% Ocupantes } \\
\text { en vivienda } \\
\text { sin drenaje } \\
\text { ni excusado }\end{array}$ & $\begin{array}{c}\text { \% Ocupantes } \\
\text { en vivienda } \\
\text { sin energía } \\
\text { eléctrica }\end{array}$ & $\begin{array}{c}\text { \% Ocupantes } \\
\text { en vivienda } \\
\text { sin agua } \\
\text { entubada }\end{array}$ & $\begin{array}{l}\text { \% Viviendas } \\
\text { con algún } \\
\text { nivel de } \\
\text { hacina- } \\
\text { miento } \\
\end{array}$ & $\begin{array}{c}\text { \% Ocupantes } \\
\text { en vivienda } \\
\text { con piso de } \\
\text { tierra }\end{array}$ & $\begin{array}{c}\% \text { población } \\
\text { en } \\
\text { localidades } \\
\text { con menos } \\
\text { de } 5000 \text { hab. }\end{array}$ & $\begin{array}{c}\text { \% población } \\
\text { ocupada con } \\
\text { ingresos de } \\
\text { hasta } 2 \\
\text { salarios } \\
\text { mínimos } \\
\end{array}$ & Entidad & $\begin{array}{c}\text { Ranking de } \\
\text { marginación } \\
\text { escala de: } \\
0 \text { a } 100\end{array}$ \\
\hline Pais México & 112336538 & 6,93 & 19,93 & 3,57 & 1,77 & 8,63 & 36,53 & 6,58 & 28,85 & 38,66 & Quiriego & 100 \\
\hline Edo. Sonora & 2662480 & 3,06 & 14,4 & 1,68 & 1,57 & 3,08 & 34,77 & 5,41 & 17,39 & 30,66 & Alamos & 70,93112552 \\
\hline Aconchi & 2637 & 4,03 & 23,43 & 1,52 & 0,3 & 0,08 & 34,52 & 1,75 & 100 & 40,13 & Rosario & 61,21660226 \\
\hline Agua Prieta & 79138 & 2,05 & 12,8 & 0,36 & 2,97 & 0,95 & 34,13 & 2,57 & 2,38 & 38,04 & Yécora & 58,97059361 \\
\hline Alamos & 25848 & 11,63 & 33,54 & 12,44 & 7,28 & 10,99 & 45,76 & 23,3 & 63,85 & 54,8 & \multicolumn{2}{|c|}{ San Miguel d 51,57228234 } \\
\hline Altar & 9049 & 4,35 & 25,38 & 3,04 & 1,01 & 4,37 & 40,72 & 1,84 & 12,4 & 25,65 & Etchojoa & 42,05769984 \\
\hline Arivechi & 1253 & 5,14 & 30,09 & 2,78 & 10,45 & 0 & 25,98 & 4,41 & 100 & 51,77 & Cucurpe & 41,8218971 \\
\hline Arizpe & 3037 & 3,77 & 27,75 & 1,92 & 3,21 & 4,34 & 20,89 & 4,11 & 100 & 50 & \multicolumn{2}{|c|}{ San Ignacio F 39,20096813 } \\
\hline Atil & 625 & 2,62 & 17,03 & 0,48 & 0,8 & 1,28 & 18,07 & 0 & 100 & 44,97 & La Colorada & 36,9455122 \\
\hline Bacadéhuachi & 1252 & 4,87 & 31,93 & 2,09 & 0,24 & 0 & 27,07 & 0,96 & 100 & 56,27 & Bacanora & 36,87343715 \\
\hline Bacanora & 784 & 7,6 & 37,68 & 4,09 & 4,61 & 1,02 & 21,83 & 5,11 & 100 & 58,33 & Trincheras & 35,28979695 \\
\hline Bacerac & 1467 & 3,94 & 23,87 & 1,57 & 3,23 & 1,93 & 28,54 & 8,88 & 100 & 67,88 & San Javier & 34,94045505 \\
\hline Bacoachi & 1646 & 3,94 & 24,23 & 1,84 & 5,7 & 3,87 & 20,89 & 2,21 & 100 & 43,77 & Bácum & 34,5483518 \\
\hline Bácum & 22821 & 6,24 & 24,29 & 5,06 & 1,89 & 2,21 & 45,39 & 7,52 & 72,65 & 53,53 & Nácori Chico & 33,81683268 \\
\hline Banámichi & 1646 & 3,04 & 19,04 & 1,35 & 1,89 & 0,55 & 27,18 & 0,68 & 100 & 35,04 & Sahuaripa & 33,54299941 \\
\hline Baviácora & 3560 & 4,16 & 26,42 & 0,54 & 0,71 & 0,62 & 27,9 & 1,78 & 100 & 35,77 & Huatabampc & o 32,46651354 \\
\hline Bavispe & 1454 & 2,72 & 24,61 & 1,03 & 0,97 & 0,83 & 21,05 & 1,17 & 100 & 60,88 & Arivechi & 31,84283006 \\
\hline Benjamín Hill & 5275 & 3,97 & 18,29 & 1,76 & 2,45 & 0,79 & 27,84 & 2,86 & 3,87 & 40,19 & Onavas & 31,59459083 \\
\hline Caborca & 81309 & 4,45 & 20,8 & 1,72 & 1,07 & 1,83 & 36,78 & 4,9 & 26,3 & 29,09 & Opodepe & 31,50463729 \\
\hline Cajeme & 409310 & 2,56 & 11,83 & 0,79 & 0,68 & 1,07 & 32,91 & 3,56 & 10,02 & 32,51 & Bacerac & 30,00338536 \\
\hline Cananea & 32936 & 1,29 & 8,6 & 0,46 & 1,02 & 0,31 & 23,18 & 1,27 & 4,18 & 28,74 & Benito Juáre & 29,54918819 \\
\hline Carbó & 5347 & 5,07 & 27,66 & 2,99 & 2,05 & 0,7 & 39,66 & 4,63 & 100 & 22,58 & Rayón & 28,62389715 \\
\hline La Colorada & 1663 & 6,55 & 38,04 & 6,07 & 8 & 6,25 & 23,5 & 3,62 & 100 & 37,36 & País México & 28,52616296 \\
\hline Cucurpe & 958 & 4,64 & 35,93 & 4,19 & 22,33 & 4,61 & 19,28 & 3,98 & 100 & 47,58 & Soyopa & 28,46220389 \\
\hline Cumpas & 6362 & 2,17 & 17,93 & 1,75 & 0,25 & 0,57 & 21,72 & 1,64 & 100 & 35,95 & Tubutama & 27,19118998 \\
\hline Divisaderos & 813 & 1,35 & 19,19 & 1,48 & 0,98 & 0,5 & 20,96 & 3,08 & 100 & 49,36 & Sáric & 27,04734791 \\
\hline Empalme & 54131 & 3,3 & 14,69 & 1,57 & 1,25 & 1,36 & 38,33 & 5,98 & 21,46 & 37,29 & \multicolumn{2}{|c|}{ San Pedro de 25,8114531} \\
\hline Etchojoa & 60717 & 6,28 & 21,21 & 3,6 & 4,43 & 1,08 & 54,47 & 16,54 & 69,94 & 57,15 & Navojoa & 24,58689777 \\
\hline Fronteras & 8639 & 2,55 & 16,42 & 1,65 & 2,27 & 1,04 & 29,22 & 3,17 & 21,88 & 22,22 & Arizpe & 23,94338195 \\
\hline Granados & 1150 & 2,42 & 20,6 & 0,61 & 0,52 & 0,52 & 18,35 & 0,78 & 100 & 39,62 & \multicolumn{2}{|c|}{ Suaqui Granc 23,76479661} \\
\hline Guaymas & 149299 & 3,51 & 14,82 & 3,76 & 2,22 & 1,08 & 35,39 & 10,76 & 13,69 & 35 & Bacadéhuact & + 23,56721728 \\
\hline Hermosillo & 784342 & 2,11 & 10,8 & 0,83 & 0,72 & 1,93 & 31,27 & 4,72 & 4,13 & 19,24 & Carbó & 22,81238186 \\
\hline Huachinera & 1350 & 3,37 & 30,58 & 3,48 & 2,15 & 0,22 & 26,65 & 2,59 & 100 & 38,88 & Mazatán & 22,2226573 \\
\hline Huásabas & 962 & 3,8 & 21,05 & 0,31 & 1,46 & 0,52 & 18,88 & 1,66 & 100 & 39,24 & Bacoachi & 21,65880251 \\
\hline Huatabampo & 79313 & 5,28 & 21,69 & 3,63 & 2,06 & 6,11 & 47,06 & 4,69 & 61,58 & 61,46 & Tepache & 21,58088702 \\
\hline Huépac & 1154 & 2,78 & 21,82 & 0,78 & 1,04 & 0 & 23,17 & 0,52 & 100 & 30,9 & Huachinera & 21,09515531 \\
\hline Imuris & 12316 & 3,57 & 19,95 & 2,64 & 2,47 & 1,09 & 35,23 & 3,78 & 44,45 & 31 & Guaymas & 20,27979431 \\
\hline Magdalena & 29707 & 2,13 & 13,96 & 1,14 & 1,42 & 2,04 & 30,01 & 3,4 & 10,44 & 17,83 & Santa Cruz & 18,51101742 \\
\hline Mazatán & 1350 & 3,82 & 27,28 & 3,88 & 3,81 & 2,97 & 19,67 & 3,12 & 100 & 38,81 & Altar & 18,05437982 \\
\hline Moctezuma & 4680 & 2,05 & 16,57 & 0,19 & 0,66 & 0,47 & 20,98 & 1 & 100 & 26,91 & Bavispe & 17,71806325 \\
\hline Naco & 6401 & 1,47 & 13,61 & 0,57 & 1,75 & 0,32 & 27,97 & 1,13 & 5,26 & 29,11 & Aconchi & 17,65393255 \\
\hline Nácori Chico & 2051 & 4,62 & 36,06 & 3,86 & 11,14 & 0,24 & 26,22 & 4,15 & 100 & 47,72 & Villa Pesque & 17,02111512 \\
\hline Nacozari de García & 12751 & 1,94 & 11,99 & 0,52 & 0,59 & 0,67 & 30,2 & 2,73 & 9,9 & 15,19 & Ures & 16,72533394 \\
\hline Navojoa & 157729 & 4,21 & 16,32 & 4,02 & 1,59 & 2,1 & 43,8 & 8,29 & 27,83 & 44,8 & Caborca & 16,72035163 \\
\hline Nogales & 220292 & 1,54 & 8,66 & 0,51 & 1,11 & 16,51 & 35,13 & 3,71 & 3,52 & 28,09 & Oquitoa & 16,63196049 \\
\hline Onavas & 399 & 4,67 & 42,52 & 1,78 & 1,02 & 0,51 & 32,11 & 1,53 & 100 & 69,44 & Imuris & 16,54827826 \\
\hline Opodepe & 2878 & 3,9 & 29,05 & 2,62 & 11,66 & 2,73 & 29,75 & 3,56 & 100 & 50,38 & Pitiquito & 16,34463365 \\
\hline Oquitoa & 443 & 2,06 & 21,87 & 1,58 & 2,48 & 5,42 & 23,26 & 2,03 & 100 & 42,38 & Baviácora & 16,22471418 \\
\hline Pitiquito & 9468 & 3,71 & 21,03 & 2,64 & 1,74 & 1,89 & 31,83 & 4,87 & 42,86 & 27,72 & Empalme & 15,34614245 \\
\hline Puerto Peñasco & 57342 & 2,7 & 14,48 & 0,69 & 4,98 & 2,16 & 34,62 & 4,73 & 1,02 & 24,74 & \multicolumn{2}{|c|}{ General Plut 15,24448796} \\
\hline Quiriego & 3356 & 15,83 & 41,52 & 24,33 & 21,44 & 18,54 & 42,42 & 12,24 & 100 & 71,91 & Benjamín Hil & | 13,96034086 \\
\hline Rayón & 1599 & 5,52 & 34,42 & 5 & 2,39 & 0,38 & 25,95 & 3,81 & 100 & 42,65 & San Felipe dı & 13,72983998 \\
\hline Rosario & 5226 & 10,89 & 38,06 & 13,19 & 6,28 & 4,2 & 41,25 & 9,84 & 100 & 63,73 & San Luis Río ( & ( 13,60494704 \\
\hline Sahuaripa & 6020 & 4,66 & 28,47 & 4,07 & 2,65 & 2,42 & 30,51 & 12,39 & 100 & 47,9 & Edo. Sonora & 13,43096083 \\
\hline San Felipe de Jesús & 396 & 2,73 & 18,8 & 1,79 & 1,78 & 1,02 & 22,83 & 0,26 & 100 & 46,28 & Divisaderos & 13,10464025 \\
\hline San Javier & 492 & 2,55 & 24,47 & 8,73 & 2,08 & 21,83 & 37,06 & 8,32 & 100 & 20,63 & Huásabas & 12,87087907 \\
\hline San Luis Río Colorado & 178380 & 3,25 & 17,52 & 0,75 & 1,86 & 1,08 & 34,89 & 4,99 & 8,26 & 35,76 & Banámichi & 12,52133124 \\
\hline San Miguel de Horcas & 8382 & 11,63 & 38,64 & 5,28 & 7,35 & 2,01 & 58,84 & 11,69 & 32,01 & 36,72 & Nogales & 11,83012187 \\
\hline San Pedro de la Cuev & 1604 & 4,79 & 33,76 & 3,63 & 1,01 & 4,14 & 17,12 & 2,63 & 100 & 50,1 & Puerto Peña & 11,4643766 \\
\hline Santa Ana & 16014 & 1,84 & 15,65 & 1,91 & 1,62 & 0,66 & 28,51 & 1,73 & 25,91 & 24,39 & Huépac & 9,992670648 \\
\hline Santa Cruz & 1998 & 2,25 & 15,41 & 0,83 & 2,47 & 7,08 & 28,92 & 3,81 & 100 & 49,34 & Cumpas & 9,98871576 \\
\hline Sáric & 2703 & 4,95 & 26,33 & 3,92 & 4,37 & 7,25 & 32,57 & 2,32 & 100 & 34,76 & Villa Hidalgo & 9,904838345 \\
\hline Soyopa & 1284 & 7,09 & 36,29 & 2,43 & 2,27 & 2,19 & 25,3 & 4,22 & 100 & 35,65 & Granados & 9,814964451 \\
\hline Suaqui Grande & 1121 & 5,43 & 34,19 & 1,88 & 3,34 & 1,79 & 28,4 & 0,36 & 100 & 37,73 & Atil & 9,764633475 \\
\hline Tepache & 1365 & 3,13 & 31,21 & 1,62 & 0,15 & 0 & 21,05 & 5,86 & 100 & 51,57 & Agua Prieta & 9,002895377 \\
\hline Trincheras & 1731 & 4,65 & 29,92 & 6,95 & 6,79 & 4,8 & 33,89 & 5,95 & 100 & 40,8 & Fronteras & 8,562921928 \\
\hline Tubutama & 1735 & 4,45 & 30,07 & 3,01 & 5,09 & 4,05 & 27,31 & 3,24 & 100 & 43,53 & Cajeme & 7,899704066 \\
\hline Ures & 9185 & 4,17 & 22,43 & 2,01 & 0,63 & 0,59 & 28,35 & 1,87 & 100 & 37,63 & Santa Ana & 6,390559993 \\
\hline Villa Hidalgo & 1738 & 3,21 & 25,24 & 0,69 & 0,4 & 0,12 & 18,91 & 0,69 & 100 & 27,58 & Moctezuma & 5,596429222 \\
\hline Villa Pesqueira & 1254 & 2,41 & 25,42 & 2,15 & 2,63 & 2,24 & 18,8 & 1,28 & 100 & 46,67 & Magdalena & 5,55722563 \\
\hline Yécora & 6046 & 10,61 & 37,16 & 3,54 & 7,95 & 16,76 & 37,13 & 15,49 & 100 & 53,08 & Hermosillo & 4,882262653 \\
\hline General Plutarco Elía: & 15652 & 3,54 & 20,22 & 1,33 & 1,86 & 1,79 & 40,24 & 3,31 & 17,91 & 31,79 & Naco & 3,576558307 \\
\hline Benito Juárez & 22009 & 5,6 & 22,24 & 3,8 & 3,02 & 1,18 & 48,28 & 5,68 & 37,43 & 50,73 & Nacozari de ' & ( 1,850242546 \\
\hline San Ignacio Río Muer & 14136 & 6,35 & 25,67 & 4,66 & 4,31 & 4,37 & 52,11 & 9,29 & 45,4 & 54,24 & Cananea & 0 \\
\hline
\end{tabular}


8870

http://revistainvestigacionacademicasinfrontera.com

Fuente: elaboración propia con datos del CONAPO.

Cuadro 5. Indicadores sobresalientes de los modelos CONEVAL-CONAPO.

\begin{tabular}{|c|c|c|c|c|c|}
\hline Clave & Entidad & $\begin{array}{l}\text { \% población } \\
\text { con ingresos } \\
<=2 \text { s.m. }\end{array}$ & \% Pobres & $\begin{array}{l}\text { \% } \\
\text { Pobreza } \\
\text { extrema }\end{array}$ & $\begin{array}{l}\% \\
\text { Personas } \\
<\text { LB }^{\text {b }}\end{array}$ \\
\hline$M x$ & País México & 38,66 & 46,30 & 10,74054 & 52 \\
\hline 26 & Edo. Sonora & 30,66 & 33,80 & 5,31459 & 40,22479 \\
\hline 26001 & Aconchi & 40,13 & 41,37396 & 5,92828 & 44,93185 \\
\hline 26002 & Agua Prieta & 38,04 & 46,17537 & 7,12832 & 53,8484 \\
\hline 26003 & Alamos & 54,8 & 65,04604 & 20,53043 & 66,449 \\
\hline 26004 & Altar & 25,65 & 47,66137 & 7,40321 & 51,75627 \\
\hline 26005 & Arivechi & 51,77 & 49,19132 & 7,78394 & 52,41516 \\
\hline 26006 & Arizpe & 50 & 49,75082 & 6,74966 & 52,5622 \\
\hline 26007 & Atil & 44,97 & 47,81666 & 3,01458 & 52,1439 \\
\hline 26008 & Bacadéhuachi & 56,27 & 54,94469 & 9,68983 & 56,9249 \\
\hline 26009 & Bacanora & 58,33 & 53,62862 & 5,85838 & 55,77513 \\
\hline 26010 & Bacerac & 67,88 & 53,26458 & 7,60641 & 54,8399 \\
\hline 26011 & Bacoachi & 43,77 & 40,73648 & 2,84368 & 43,05189 \\
\hline 26012 & Bácum & 53,53 & 44,47975 & 6,69713 & 51,14087 \\
\hline 26013 & Banámichi & 35,04 & 41,30977 & 4,95824 & 44,9003 \\
\hline 26014 & Baviácora & 35,77 & 40,12623 & 3,93304 & 44,58396 \\
\hline 26015 & Bavispe & 60,88 & 56,35588 & 9,37126 & 57,50856 \\
\hline 26016 & Benjamín Hill & 40,19 & 39,93397 & 5,31482 & 49,96708 \\
\hline 26017 & Caborca & 29,09 & 38,69288 & 5,45808 & 45,48753 \\
\hline 26018 & Cajeme & 32,51 & 26,57808 & 2,41272 & 34,03719 \\
\hline 26019 & Cananea & 28,74 & 39,64784 & 3,02366 & 46,71943 \\
\hline 26020 & Carbó & 22,58 & 53,03979 & 12,22898 & 57,48833 \\
\hline 26021 & La Colorada & 37,36 & 50,18791 & 7,3898 & 53,21991 \\
\hline 26022 & Cucurpe & 47,58 & 50,61033 & 8,65681 & 52,81921 \\
\hline 26023 & Cumpas & 35,95 & 34,5089 & 2,17468 & 41,67738 \\
\hline 26024 & Divisaderos & 49,36 & 42,27519 & 4,21586 & 46,25935 \\
\hline 26025 & Empalme & 37,29 & 33,43988 & 5,04903 & 40,7569 \\
\hline 26026 & Etchojoa & 57,15 & 57,64013 & 18,55399 & 61,1235 \\
\hline 26027 & Fronteras & 22,22 & 40,91985 & 6,50221 & 56,28408 \\
\hline 26028 & Granados & 39,62 & 36,02652 & 2,50048 & 39,4018 \\
\hline 26029 & Guaymas & 35 & 28,88106 & 5,56968 & 36,32839 \\
\hline 26030 & Hermosillo & 19,24 & 25,36531 & 3,01415 & 32,21639 \\
\hline 26031 & Huachinera & 38,88 & 51,82311 & 10,89866 & 52,80105 \\
\hline
\end{tabular}


Revista de Investigación

Año 10.

Académica sin Frontera

Núm. 26

ISSN: 2007.

8870

http://revistainvestigacionacademicasinfrontera.com

\begin{tabular}{|l|l|l|l|l|l|}
\hline 26032 & Huásabas & 39,24 & 37,01511 & 3,75828 & 41,29103 \\
\hline 26033 & Huatabampo & 61,46 & 52,71576 & 14,8548 & 56,69256 \\
\hline 26034 & Huépac & 30,9 & 26,68619 & 0,62026 & 32,17305 \\
\hline 26035 & Imuris & 31 & 43,95131 & 5,65016 & 47,86432 \\
\hline 26036 & Magdalena & 17,83 & 40,77855 & 4,77685 & 48,72885 \\
\hline 26037 & Mazatán & 38,81 & 38,0686 & 3,85947 & 41,01057 \\
\hline 26038 & Moctezuma & 26,91 & 34,38972 & 1,52267 & 41,47078 \\
\hline
\end{tabular}

Continuación del cuadro 5. Indicadores sobresalientes de los modelos CONEVAL-

CONAPO

\begin{tabular}{|l|l|l|l|l|l|}
\hline Clave & Entidad & $\begin{array}{l}\text { \% población } \\
\text { con ingresos } \\
<=\mathbf{2} \text { s.m. }\end{array}$ & \% Pobres & $\begin{array}{l}\text { \% } \\
\text { Pobreza } \\
\text { extrema }\end{array}$ & $\begin{array}{l}\text { \% } \\
\text { Personas } \\
\text { LB }^{\text {b }}\end{array}$ \\
\hline 26039 & Naco & 29,11 & 50,84234 & 5,88566 & 55,00243 \\
\hline 26040 & Nácori Chico & 47,72 & 61,35772 & 11,44528 & 62,78122 \\
\hline 26041 & Nacozari de García & 15,19 & 26,50975 & 3,59133 & 39,68322 \\
\hline 26042 & Navojoa & 44,8 & 34,81539 & 6,25667 & 40,23097 \\
\hline 26043 & Nogales & 28,09 & 34,09623 & 5,1455 & 41,11509 \\
\hline 26044 & Onavas & 69,44 & 56,12226 & 8,01419 & 59,8273 \\
\hline 26045 & Opodepe & 50,38 & 52,00742 & 10,66092 & 55,13851 \\
\hline 26046 & Oquitoa & 42,38 & 37,02142 & 4,2988 & 41,79606 \\
\hline 26047 & Pitiquito & 27,72 & 36,16884 & 5,12786 & 42,58277 \\
\hline 26048 & Puerto Peñasco & 24,74 & 43,74548 & 7,31611 & 50,20785 \\
\hline 26049 & Quiriego & 71,91 & 41,71677 & 11,67986 & 42,72651 \\
\hline 26050 & Rayón & 42,65 & 52,05863 & 7,39357 & 54,31409 \\
\hline 26051 & Rosario & 63,73 & 61,28995 & 15,20936 & 66,52679 \\
\hline 26052 & Sahuaripa & 47,9 & 54,72261 & 10,3594 & 59,52646 \\
\hline 26053 & San Felipe de Jesús & 46,28 & 35,63483 & 1,98937 & 40,07316 \\
\hline 26054 & San Javier & 20,63 & 30,25246 & 5,68051 & 31,96876 \\
\hline 26055 & San Luis Río Colorado & 35,76 & 38,75765 & 4,91837 & 43,26527 \\
\hline 26056 & San Miguel de Horcasitas & 36,72 & 71,2382 & 28,5542 & 73,73399 \\
\hline 26057 & San Pedro de la Cueva & 50,1 & 45,2548 & 5,63532 & 47,37623 \\
\hline 26058 & Santa Ana & 24,39 & 36,15361 & 3,50949 & 42,46777 \\
\hline 26059 & Santa Cruz & 49,34 & 52,65552 & 10,35492 & 54,05378 \\
\hline 26060 & Sáric & 34,76 & 53,68657 & 9,67527 & 54,24547 \\
\hline 26061 & Soyopa & 35,65 & 49,36149 & 6,03745 & 52,96443 \\
\hline 26062 & Suaqui Grande & 37,73 & 51,5776 & 6,67023 & 54,52072 \\
\hline 26063 & Tepache & 51,57 & 56,4022 & 6,50088 & 58,75585 \\
\hline & & & & \\
\hline
\end{tabular}


Año 10.

Académica sin Frontera

Núm. 26

ISSN: 2007

8870

http://revistainvestigacionacademicasinfrontera.com

\begin{tabular}{|l|l|l|l|l|l|}
\hline 26064 & Trincheras & 40,8 & 55,61136 & 10,06168 & 58,70721 \\
\hline 26065 & Tubutama & 43,53 & 63,15068 & 9,02676 & 64,36518 \\
\hline 26066 & Ures & 37,63 & 41,46607 & 3,89202 & 46,73099 \\
\hline 26067 & Villa Hidalgo & 27,58 & 33,05396 & 2,36722 & 39,34665 \\
\hline 26068 & Villa Pesqueira & 46,67 & 47,26133 & 3,33986 & 49,87342 \\
\hline 26069 & Yécora & 53,08 & 78,74554 & 26,58528 & 80,47098 \\
\hline 26070 & $\begin{array}{l}\text { General Plutarco Elías } \\
\text { Calles }\end{array}$ & 31,79 & 53,34801 & 16,52739 & 56,80836 \\
\hline 26071 & Benito Juárez & 50,73 & 59,59379 & 18,08271 & 63,51594 \\
\hline 26072 & San Ignacio Río Muerto & 54,24 & 59,37263 & 17,23545 & 63,43096 \\
\hline
\end{tabular}

ase refiere al \% de la población con ingresos menores a dos salarios mínimos.

bSe refiere al \% de la población por debajo de la línea de bienestar.

Fuente: elaboración propia con datos del CONAPO y CONEVAL 


\section{http://revistainvestigacionacademicasinfrontera.com}

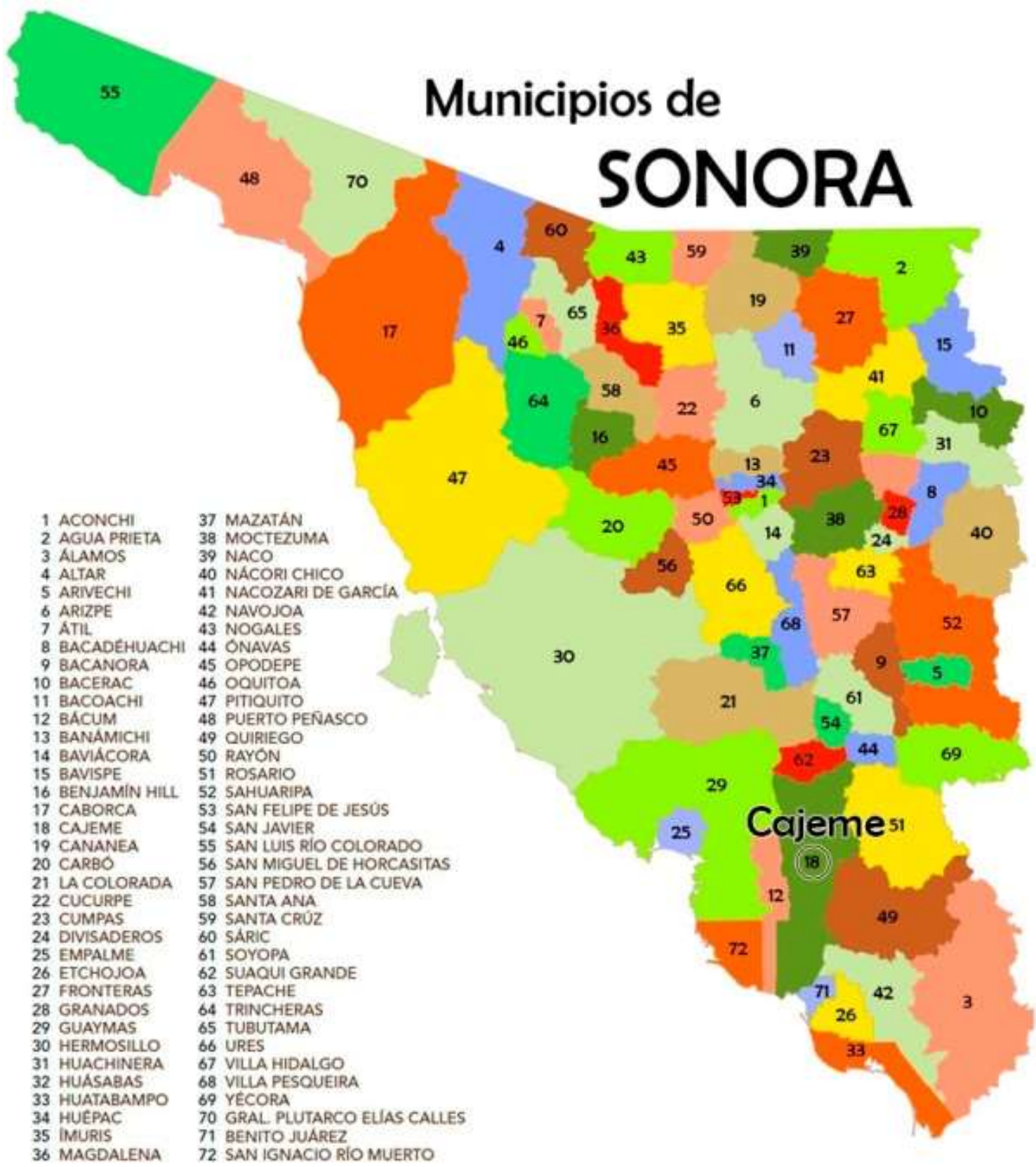

Fuente:

https://www.google.com.mx/search?q=MAPA+DE+LOS+MUNICIPIOS+DEL+ESTADO+DE+SONORA\&tbm=isch\&imgil=tUkh CzcUUlTwAM\%253A\%253Bj5Id7R8f1FxYPM\%253Bhttps\%25253A\%25252F\%25252Fes. wikipedia.org\%25252Fwiki\%25252F Anexo\%25253AMunicipios_de_Sonora\&source=iu\&pf=m\&fir=tUkhCzcUUITwAM\%253A\%252Cj5Id7R8f1FxYPM\%252C_\&us $\mathrm{g}=\_$S5ue_ouPJncfyNr7cgxNf7NvgoA\%3D\&biw=1366\&bih=662\&ved=0ahUKEwiAobbQ0tzTAhVGyoMKHS0JBckQyjcINQ\&ei $=\mathrm{U} 3 \mathrm{oOWcCrIsaUjwStkpTIDA \# imgrc=}=\mathrm{coALwEDOjjG2vM:}$ 